\section{Neurological symptoms due to Coronavirus disease 2019}

\author{
Farage Ftiha, ${ }^{1}$ Moshe Shalom, ${ }^{2}$ \\ Henry Jradeh 1 \\ 1CUNY Brooklyn College, NY; ${ }^{2}$ Sackler \\ School of Medicine, NY, USA
}

\begin{abstract}
In this review, we focus on summarizing everything that is known about the neurological effects of Severe Acute Respiratory Syndrome Coronavirus 2 (SARS-COV-2). It has been shown that Coronavirus Disease 2019 (Covid-19) may result in neuromuscular disorders or damage to nerves outside of the brain and spinal cord, which may lead to weakness, numbness, and pain. Published literature has stated that SARS-COV-1 may infect the central nervous system and due to its similarities to SARS-COV-2, we suspect that SARS-COV-2 has the same potential. We conclude that Covid-19 has neurological manifestations. Further research should be done in this field to understand the full extent of this virus.
\end{abstract}

\section{Introduction}

In December of 2019, Severe Acute Respiratory Syndrome Coronavirus 2 (SARS-COV-2) emerged in Wuhan, China, and rapidly spread across many other countries. ${ }^{1}$ Early studies reported a possible link from a wild animal market for most cases of infection, indicating the likelihood of it being zoonotic in nature. It is suspected that the reservoir for SARS-COV-2 is a Chinese horseshoe bat, however further studies have indicated a human-to-human transmission model of SARS-COV-2 through droplets or direct contact is the most likely method of transmission amongst most patients. ${ }^{2-5}$ With the interconnected nature of our globalized society, travel has become a very prevalent epidemiological link in disease transmission, especially during the current pandemic. On March 11, 2020, the World Health Organization officially classified Coronavirus Disease 2019 (COVID-19) as a pandemic. ${ }^{1}$ People with prolonged contact with an infected individual are at high risk of contracting the virus. To gain more accurate data, it is useful to use an electronic literature search to gather data from published findings and case studies. ${ }^{6}$

In this paper, we review literature concerned with the neurological implications of
Coronavirus Disease 2019 (Covid-19) in hopes of shedding light on potentially overlooked, yet significant, neurologic complications of the virus. Published literature was compiled using Pubmed and Google Scholar as search engines and Sars Neurology, Sars Cov 2 Neurology as keywords used in the search. Studies lacking the focus on neurology were excluded in our search. Very little information is known about how Severe Acute Respiratory Syndrome Coronavirus 2 (Sars-Cov-2) interacts with the central and peripheral nervous system, and new information about the virus is being added to the literature every day. Concerns of neurological complications in patients suffering from Covid-19 were raised when research reports surfaced from Wuhan, China, suggesting that neurologic symptoms are apparent in $36.4 \%$ of patients and are more common in patients with severe infection (45.5\%). ${ }^{7}$ We compare this newfound data of SARS-COV-2 to the limited amount of published literature that has surfaced in regards to the neurological complications of SARS-COV-1 in hopes to advance the knowledge of the scientific community on the neurological pathology of SARS-COV-2.

\section{Pathology of SARS-COV-2}

The family of Coronaviridae is singlestranded, positive-sense RNA viruses that are surrounded by a viral envelope. The suborder Cornidovineae is split up into alpha coronavirus, beta coronavirus, gamma coronavirus, and delta coronavirus. ${ }^{8}$ Most coronaviruses share mainly similar genomes and the open reading frames 1a and $1 \mathrm{~b}$ compromise almost two-thirds of the genome. The remaining portion of the genome is responsible for encoding structural proteins such as the nucleocapsid, envelope, and membrane proteins. ${ }^{1,6} \mathrm{SARS}-\mathrm{COV}-2$ is enveloped by a lipid bilayer that contains viral fusion proteins that aid with the fusion of viruses to the cell. When SARS-COV-2 injects its genome into the cell, the virus hijacks the host's ribosomes for protein production with the help of its viral RNA 5' cap and a poly-A tail.6,9 The first protein produced by the viral positive-sense RNA is an RNA Dependent RNA Polymerase (RDRP). RDRP is a replicase and it is responsible for transcribing a negative-sense RNA strand by reading the original positive sense RNA strand from the 5' to the 3' end. Once the negative-sense RNA strand is complete, the RDRP reads the negative-sense RNA strands from the 3 ' to the 5' end to produce another positive sense RNA strand. 4
Correspondence: Farage Ftiha, CUNY Brooklyn College, 2900 Bedford Ave, Brooklyn, NY 11210, USA.

E-mail: farageftiha123@gmail.com

Key words: SARS-COV-2, Covid-19, Neurological effects.

Contributions: The authors contributed equally.

Conflict of interest: The authors declare no potential conflict of interest.

Funding: none.

Availability of data and materials: All Materials were found online using PubMed.

Ethics approval and consent to participate: No experiment was directly conducted, so no ethics approval nor consent is necessary.

Informed consent: Not necessary.

Received for publication: 21 May 2020.

Accepted for publication: 28 MAy 2020.

This work is licensed under a Creative Commons Attribution NonCommercial 4.0 License (CC BY-NC 4.0).

CCopyright: the Author(s), 2019

Licensee PAGEPress, Italy

Neurology International 2020; 12:8639

doi:10.4081/ni.2020.8639

\section{Attachment of SARS-COV-2 to ACE2 receptors}

SARS-COV-2 utilizes angiotensin-converting enzyme 2 (ACE2) as an entry point in the early stages of infection. It does this by attaching its spike S1 protein to ACE2's enzymatic domain, which causes the cell to undergo endocytosis. After this occurs, there is a down-regulation of surface ACE2 proteins, which leads to an increased level of angiotensin II. The virus also requires that the $\mathrm{S}$ protein is primed by serine protease TMPRSS2, a protein on the host cell. 10 The $\mathrm{S}$ protein is a class I viral fusion protein, which means that it is a trimer in the fusion states, relies on proteolytic cleavage for activation and contains alpha-helices and heptad repeats (HR) regions. ${ }^{11}$ The organs most affected by SARS-COV-2 are the lungs. The virus accesses the host cells through the enzyme angiotensin-converting enzyme 2 (ACE 2) which is abundant in type II alveolar cells in the lungs. ${ }^{6}$ Furthermore, it has been shown that glial cells and neurons of the central nervous system express ACE 2 receptors as well, making nervous tissue a possible infection site for SARS-COV-2.9 


\section{Zinc as a countermeasure}

It has been shown that Zinc $2+$ ion is able to inhibit coronavirus replication in vitro, with a direct relationship between the increased intracellular concentration of $\mathrm{Zn}$ $2+$ and a decrease in the viral byproduct of RDRP. 10

Charged ions such as $\mathrm{Zn} \mathrm{2+}$ are unable to pass the hydrophobic part of the cell membrane through simple diffusion, requiring the use of a membrane ionophore. Pyrithione, one ionophore that exists in the body, provides a mechanism to allow the Zinc to enter ions into the cytosol of the cell. 12

This may help in the resistance of the spread infection from SARS-COV-2,13 however it has not been found how increased levels of zinc can aid in preventing or combating the suspected neurological symptoms of SARS-COV-2. More research should be done to reveal how we can use zinc to sabotage viral reproduction in order to prevent or treat neurological symptoms of SARS-COV-2.

\section{The role of Zinc in acute immune response and neurologi- cal complications}

It is known that zinc is a major trace element found in the central nervous system, and plays a crucial role in proper neurological functioning. Zinc deficiency has been found to be related to oxidative stress as well as impairments in olfaction and gustation, ${ }^{14}$ both of which have been observed in SARS-COV-2 patients.

Zinc has also been found to be a major factor in the human body's acute immune response. Zinc floods into cellular compartments of vital organs, especially the liver, causing transient hypozincemia in the host's serum during early responses to infection. This zinc redistribution also creates an unfavorable environment for microbial pathogens, who need zinc to survive. ${ }^{15,16}$

One possible complication of SARSCOV-2 is the potential for cytokine release syndrome, characterized by a rapid cytokine storm. ${ }^{17}$ This rapid increase in cytokines, such as Interleukin-6 (IL-6), is attributed to the immune system's response to viral infection by SARS-COV-2. Cytokine storms can cause complications including respiratory failure and neurological disorders. Zinc deficiency promotes IL-6 production, along with other immune markers. Even more so, zinc deficiency can promote immune cell activation and a pro-inflammatory response. 11
Furthermore, cytokine release syndrome can result in acute necrotizing encephalopathy (ANE), a rare hemorrhagic encephalopathy related to viral infection and cytokine release syndrome. This condition has been observed in influenza patients as well as a small number of SARS-COV-2 patients. ${ }^{18}$ Viral particles do not require a direct invasion the central nervous system for ANE to develop, however. A breakdown of the blood-brain barrier, seizures, and strokes are complications ANE.

Subsequently, neuroinflammation can occur as a result of the cytokine storm, specifically as a result of IL-6. IL-6 further activates the production of other cytokines, which worsens the encephalopathy in a positive feedback-like manner. ${ }^{11}$

\section{Neurological symptoms of SARS- $\mathrm{COV}-1$}

Towards the end of 2002 a coronavirus disease emerged in China, similar to SARSCOV-2, and spread throughout parts of Asia. The virus was named SARS-COV, now denoted as SARS-COV-1.1 SARSCOV-1 has a high mortality rate in patients older than the age of 65 , at approximately $50 \% .{ }^{19}$ A limited amount of published literature has surfaced in regards to the neurological complications of SARS-COV-1 and it has been reported that these complications appear 2-3 weeks into the course of the illness. The neurological complications include but are not limited to axonal peripheral neuropathy and myopathy. ${ }^{19}$ Furthermore, it has been reported that patients with SARS-COV-1 also had olfactory neuropathy with an onset of three weeks into the illness, ${ }^{5}$ similar to reported symptoms of SARS-COV-2. Alteration in smell and taste were frequently reported by mildly symptomatic patients of SARSCOV-2 infection and were often the first apparent symptom of the disease. ${ }^{2}$ These results must be interpreted with caution, however, due to the limitations of the study that has made these findings. A doubleblind study would be best to determine such results and this study is not the gold standard in medicine. When transgenic mice were infected intranasally, researchers discovered the virus residing in the thalamus and brainstem, suggesting that the neural pathway of viral particles originated in the olfactory nerve. ${ }^{7}$

After the SARS-COV-1 outbreak there had been several proposed mechanisms of neurological manifestations in SARS-COV1 patients. ${ }^{17}$ The different neurological symptoms observed in this study were poly- neuropathy, and rhabdomyolysis, which may result from systemic inflammatory response syndrome. ${ }^{19,20}$ The pathogenetic role of a direct attack of SARS-COV-1 on the peripheral nerves and muscles was suspected in this report. ${ }^{17}$ Multiple factors of cerebral infarction in SARS-COV-1 patients included: hypercoagulable status related to both SARS-COV-1 and the usage of intravenous immunoglobulin, septic and cardiogenic shock, and possible viral inflammation of blood vessels. ${ }^{17}$ Because of the limited number of reported cases of SARS-COV-1 patients with neurological symptoms, the clear pathogenesis of the neurological problems in SARS-COV-1 is not well understood.

Furthermore, there have been several case studies during the SARS-COV-1 outbreak that reported patients experiencing fever, chills, productive cough, and diarrhea. In one particular case study, a patient began to vomit, and experience episodes of four-limb twitching approximately one week after hospital admission. Within a few hours, the patient became confused and disorientated. ${ }^{21}$ The researchers performed further virologic investigations due to the presentation of the seizures. The researchers decided to analyze the extracted RNA from the cerebrospinal fluid (CSF) and serum samples of the patient by real-time quantitative RT-PCR assay targeting the polymerase region of the SARS-COV-1 genome. ${ }^{21}$ The researchers found that the SARS-COV-1 RNA was present in both the CSF and the serum, with viral loads of 6884 and 6750 copies/mL, respectively. ${ }^{21}$ These results suggest meningitis infection by SARSCOV-1.

\section{Neurological symptoms of SARS- COV-2}

One report surfacing from Wuhan, China indicates that neurologic symptoms were seen in $36.4 \%$ of patients with SARSCOV-2, and were more common in patients with severe infection $(45.5 \%){ }^{7}$ The neurological symptoms discussed were myopathy, loss of sense of smell or taste, and stroke. More nonspecific symptoms were also implicated in the study such as lower level of consciousness, headaches, dizziness, and seizure. ${ }^{7}$

These symptoms are grouped into several categories, including acute cerebrovascular disease-related symptoms, intracranial infection-related symptoms, peripheral nervous system symptoms, and neuromuscular symptoms. ${ }^{22}$ With regards to headaches, one study reported that $8 \%$ of patients 
experienced them, and another study reported that $34 \%$ of patients complained of headaches. ${ }^{12,19}$

Among confirmed patients with COVID-19, a significant number of severely affected individuals were elderly. High D-dimer levels were observed in COVID19 patients in one intensive care unit (ICU), predisposing them to a hypercoagulable state. 22 A lower platelet count in critical cases, as found by the same study, suggests that patients with hypertension who are infected by SARS-CoV-2 may experience increased blood pressure and are at a higher risk for cerebrovascular hemorrhage, in addition to the fact that elderly patients are already a high-risk group for developing cerebrovascular disease. 22

Moreover, intracranial infection-related symptoms such as headache, epilepsy, and disturbance of consciousness have been reported by doctors observing these symptoms in COVID-19 patients. ${ }^{18}$ Past evidence from SARS-COV-1 revealed a possible central nervous system infection for coronaviruses, and given the similar genetic sequences of SARS-COV-1 and SARSCOV-2, and their use of the same ACE2 receptor in order to gain entry to host cells, neurologists should consider the possibility of central nervous system infection by SARS-CoV-2.18,22

A case study with 214 patients with COVID 19 revealed that $78(36.4 \%)$ suffered from neurological symptoms. 7 The researchers and neurologists analyzed the data and categorized the symptoms into three categories. If the central nervous system had been affected, the patients developed symptoms such as dizziness, headache, impaired consciousness, acute cerebrovascular disease, ataxia, and seizure. Patients with affected peripheral nervous system had clinical manifestations including taste, smell, and vision impairment as well as nerve pain. ${ }^{7}$ The final category was for patients who suffered sustained injury to their skeletal-muscular system. Patients who had a more severe infection, as well as those who were older and had other underlying disorders such as hypertension, were more likely to have these neurological symptoms. Patients with more severe infection were $5.1 \%$ more likely to have acute cerebrovascular diseases, $12.4 \%$ more likely to have impaired consciousness, and 14.5\% more likely to have a skeletal muscle injury. ${ }^{7}$

Recent literature has reported cases of Guillain-Barré Syndrome (GBS) as a complication of the novel coronavirus. ${ }^{8,23}$ GBS is a polyneuropathy that commonly develops post infection. Immune attack of nerve cells causes the typical tingling in the extre- mities that is commonly reported as being the first symptom of GBS. Little is known about the interaction between SARS-COV2 infection and GBS, however clinicians should be aware of the possibility of GBS complication when treating patients tested positive for SARS-COV-2.

\section{Discussion and conclusions}

Much of current knowledge of the SARS-COV-2 virus revolves around its relatedness to SARS-COV-1. Due to the understanding that most coronaviruses share mainly similar genomes and the open reading frames, $1 \mathrm{a}$ and $1 \mathrm{~b}$, comprise almost two-thirds of the genome, researchers can look into previously published research reports of the SARS-COV-1 outbreak in the early 2000's in order to predict various implications of SARS-COV-2. This is vital to understanding overlooked avenues of research such as neurological implications of SARS-COV-2.

SARS-COV-2 utilizes angiotensin-converting enzyme 2 (ACE2) as an entry point in the early stages of infection, as seen in previous studies. It has been shown that glial cells and neurons of the central nervous system express ACE 2 receptors, ${ }^{9}$ and that the central nervous system is capable of invasion by SARS-COV-1 in a rat model. ${ }^{7}$ This effectively makes nervous tissue a possible target of infection by the novel coronavirus.

Zinc $2+$ ion is able to inhibit coronavirus replication in vitro, however, the mechanism with which increased zinc levels can aid in preventing or combating the suspected neurological symptoms of SARS-COV2 is still unknown. More research should be done in this avenue to determine how to treat neurological symptoms of SARSCOV-2, and zinc supplementation should be considered.

Furthermore, one possible complication of SARS-COV-2 is the potential for cytokine release syndrome, characterized by rapid cytokine storms. ${ }^{17}$ This rapid increase in cytokines is attributed to the immune system's response to viral infection by SARSCOV-2. Cytokine storms can cause complications including respiratory failure and neurological disorders. Zinc deficiency promotes cytokine production, along with other immune markers, and therefore can lead to further complications. Zinc deficiency has also been found to be related to oxidative stress as well as impairments in olfaction and gustation, ${ }^{14}$ both of which have been observed in SARS-COV-2 patients, adding another possible benefit of zinc supplementation.
A limited amount of published literature has surfaced in regards to the neurological complications of SARS-COV-1. However, it has been reported that these complications appear 2-3 weeks into the course of the illness and the reported neurological complications include axonal peripheral neuropathy and myopathy. ${ }^{19}$ Interestingly, it has also been reported that patients with SARS-COV-1 also had olfactory neuropathy with an onset of three weeks into the illness, similar to patients suffering with SARS-COV-2. Different neurological symptoms observed in the SARS-COV-1 outbreak were polyneuropathy and rhabdomyolysis, which may result from systemic inflammatory response syndrome. Researchers suspect a pathogenetic role of a direct attack of SARS-COV-1 on the peripheral nerves and muscles. Finally, researchers found that the SARS-COV-1 RNA was present in both the CSF and the serum of infected patients. This leads us to believe that the virus has the capability to cause a meningitis infection due to the observation of viral loads in CSF of several patients. Clinicians should be aware of this complication.

Reports surfaced from Wuhan, China indicating that clinicians observed neurologic symptoms in $36.4 \%$ of patients with SARS-COV-2. The neurological symptoms discussed were myopathy, loss of sense of smell or taste, and stroke. These observed symptoms were similar to that observed in the SARS-COV-1 outbreak, indicating yet again a correlation between SARS-COV-1 and SARS-COV-2. More nonspecific symptoms were also implicated in the reports that surfaced from Wuhan, including depressed consciousness, headaches, dizziness, and seizures. With regards to headaches, one study reported that $8 \%$ of patients experienced them, and another study reported that $34 \%$ of patients complained of headaches. A significant number of severely affected individuals had high D-dimer levels predisposing the individuals to a hypercoagulable state. A lower platelet count in critical cases suggests that patients with hypertension who are infected with SARS-CoV-2 may experience increased blood pressure and are at a higher risk for cerebrovascular hemorrhage. Past evidence from SARS-COV-1 revealed a possible central nervous system infection for coronaviruses, and given the similar genetic sequences of SARS-COV-1 and SARS-COV-2, especially the similarity in their use of the same ACE2 receptor in order to gain entry to host cells, neurologists should consider the possibility of central nervous system infection by SARS-CoV-2. Lastly, recent literature reported cases of Guillain-Barré 
Syndrome (GBS) as a complication of the novel coronavirus post infection.

Knowledge on the novel coronavirus is surfacing every day, and as time continues our understanding of how to combat the current pandemic increases. We write this review with the goal of enriching the knowledge of physicians and other healthcare workers who are treating patients infected with the virus, and hope that unforeseen neurological complications in such patients can be avoided.

\section{References}

1. Lai CC, Shih TP, Ko WC, et al. Severe acute respiratory syndrome coronavirus 2 (SARS-CoV-2) and coronavirus disease-2019 (COVID-19): The epidemic and the challenges. Int $\mathrm{J}$ Antimicrobi Agents 2020;55.

2. Basso LGM, Vicente EF, Crusca E, et al. SARS-CoV fusion peptides induce membrane surface ordering and curvature. Sci Rep 2016;6:37131.

3. Engel WK. Intravenous immunoglobulin $\mathrm{G}$ is remarkably beneficial in chronic immune dysschwannian/dysneuronal polyneuropathy, diabetes- 2 neuropathy, and potentially in severe acute respiratory syndrome. In Acta Myologica 2003;22:97-103.

4. Graham Carlos W, Dela Cruz CS, Cao B, et al. Novel Wuhan (2019-NCoV) coronavirus. Am J Respir Crit Care Med 2020;201:7-8.

5. Hwang CS. Olfactory neuropathy in severe acute respiratory syndrome: Report of a case. Acta Neurol Taiwanica 2006;15:26-8.

6. Sellner J, Taba P, Öztürk S, Helbok R. The need for neurologists in the care of
COVID-19 patients. Eur J Neurol 2020.

7. Mao L, Jin H, Wang $M$, et al. Neurologic Manifestations of Hospitalized Patients with Coronavirus Disease 2019 in Wuhan, China. JAMA Neurol 2020.

8. Coen M, Jeanson G, Alejandro Culebras Almeida L, et al. Guillain-Barré Syndrome as a Complication of SARSCoV-2 Infection. Brain Behav Immun 2020 [Online ahead of print].

9. Baig AM, Khaleeq A. Ali U, Syeda H. Evidence of the COVID-19 Virus Targeting the CNS: Tissue Distribution, Host-Virus Interaction, and Proposed Neurotropic Mechanisms. ACS Chem. Neurosci 2020;7:995-8.

10. te Velthuis AJW, Arnold JJ, Cameron CE. The RNA polymerase activity of SARS-coronavirus nsp12 is primer dependent. Nucleic Acids Res 2010;38: 203-14.

11. Wong CP, Rinaldi NA, Ho E. Zinc deficiency enhanced inflammatory response by increasing immune cell activation and inducing IL6 promoter demethylation. Mol Nutr Food Res 2015;59991-9.

12. Huang C, Wang Y, Li X, et al. Clinical features of patients infected with 2019 novel coronavirus in Wuhan, China. The Lancet 2020;395:497-506.

13. te Velthuis AJW, van den Worm SHE, Sims AC, et al. $\mathrm{Zn} 2+$ Inhibits Coronavirus and Arterivirus RNA Polymerase Activity In Vitro and Zinc Ionophores Block the Replication of These Viruses in Cell Culture. PLoS Pathogens 2010;6:e1001176.

14. Pisano M, Hilas O. Zinc and Taste Disturbances in Older Adults: A Review of the Literature. Consultant Pharmacist 2016;31:267-70

15. Wessels I, Maywald M, Rink, L. Zinc as a gatekeeper of immune function.
Nutrients 2017;9.

16. Besecker BY, Exline MC, Hollyfield J, et al. A comparison of zinc metabolism, inflammation, and disease severity in critically ill infected and noninfected adults early after intensive care unit admission. Am J Clin Nutr 2011;9: 1356-64.

17. Zhang C, Wu Z, Li JW,et al. The cytokine release syndrome (CRS) of severe COVID-19 and Interleukin-6 receptor (IL-6R) antagonist Tocilizumab may be the key to reduce the mortality. Int $\mathrm{J}$ Antimicrob Agents 2020.

18. Poyiadji N, Shahin G, Noujaim D, et al. COVID-19-associated Acute Hemorrhagic Necrotizing Encephalopathy: CT and MRI Features. Radiology 2020;201187.

19. Xu XW, Wu XX, Jiang XG, et al. Clinical findings in a group of patients infected with the 2019 novel coronavirus (SARS-Cov-2) outside of Wuhan, China: Retrospective case series. BMJ 2020;368.

20. Tsai LK, Hsieh ST, Chao CC, et al. Neuromuscular disorders in severe acute respiratory syndrome. Arch Neurol 2004;61:1669-73.

21. Hung ECW, Chim SSC, Chan PKS, et al. Detection of SARS Coronavirus RNA in the Cerebrospinal Fluid of a Patient with Severe Acute Respiratory Syndrome. Clin Chem 2003;49;:21089).

22. Liu K, Pan M, Xiao Z, Xu X. Neurological manifestations of the coronavirus (SARS-CoV-2) pandemic 2019-2020. J Neurol Neurosurg Psychiatry 2020.

23. Otmani H, El Moutawakil B, El Rafai, et al. Covid-19 and Guillain-Barré syndrome: More than a coincidence! Revue Neurologique 2020. 